\title{
Microbiology Specimen Assessment Reason Not Done
}

National Cancer Institute

\section{Source}

National Cancer Institute. Microbiology Specimen Assessment Reason Not Done. NCI

Thesaurus. Code C87898.

The explanation given as to why microbiology specimen was not assessed. 